\title{
VirSorter: mining viral signal from microbial genomic data
}

Simon Roux, Francois Enault, Bonnie L Hurwitz, Matthew B Sullivan

Viruses of microbes impact all ecosystems where microbes drive key energy and substrate transformations including the oceans, humans and industrial fermenters. However, despite this recognized importance, our understanding of viral diversity and impacts remains limited by too few model systems and reference genomes. One way to fill these gaps in our knowledge of viral diversity is through the detection of viral signal in microbial genomic data. While multiple approaches have been developed and applied for the detection of prophages (viral genomes integrated in a microbial genome), new types of microbial genomic data are emerging that are more fragmented and larger scale, such as Single-cell Amplified Genomes (SAGs) of uncultivated organisms or genomic fragments assembled from metagenomic sequencing. Here, we present VirSorter, a tool designed to detect viral signal in these different types of microbial sequence data in both a referencedependent and reference-independent manner, leveraging probabilistic models and extensive virome data to maximize detection of novel viruses. Performance testing shows that VirSorter's prophage prediction capability compares to that of available prophage predictors for complete genomes, but is superior in predicting viral sequences outside of a host genome (i.e. from extrachromosomal prophages, lytic infections, or partially assembled prophages). Furthermore, VirSorter outperforms existing tools for fragmented genomic and metagenomic datasets, and can identify viral signal in assembled sequence (contigs) as short as 3kb, while providing near-perfect identification (>95\% Recall and $100 \%$ Precision) on contigs of at least $10 \mathrm{~kb}$. Because VirSorter scales to large datasets, it can also be used in "reverse" to more confidently identify viral sequence in viral metagenomes by sorting away cellular DNA whether derived from gene transfer agents, generalized transduction or contamination. Finally, VirSorter is made available through the iPlant Cyberinfrastructure that provides a web-based user interface interconnected with the required computing resources. VirSorter thus complements existing prophage prediction softwares to better leverage fragmented, SAG and metagenomic datasets in a way that will scale to modern sequencing. Given these features, VirSorter should enable the discovery of new viruses in microbial datasets, and further our understanding of uncultivated viral communities across diverse ecosystems. 
6 Affiliations

$7 \quad{ }^{1}$ Ecology and Evolutionary Biology, University of Arizona, USA

$8 \quad{ }^{2}$ Clermont Université, Université Blaise Pascal, Laboratoire “ Microorganismes: Génome et

9 Environnement ", Clermont-Ferrand, France

$10{ }^{3}$ CNRS UMR 6023, LMGE, Aubière, France

$11{ }^{4}$ Department of Agricultural and Biosystems Engineering, University of Arizona, USA

12

13 Corresponding authors:

14

15 Matthew B Sullivan

16 University of Arizona

$17 \quad 1007$ E. Lowell St.

18 Tucson, AZ 85721

$19 \quad 520.626 .6297$

20 mbsulli@email.arizona.edu 
Viruses of microbes, mainly infecting bacteria and archaea, are ubiquitous and abundant in every type of biome sampled thus far, where virus-host interactions alter ecosystem function ranging from geochemical cycling to human health (Fuhrman, 1999; Wommack \& Colwell, 2000; Weinbauer, 2004; Breitbart \& Rohwer, 2005; Edwards \& Rohwer, 2005; Suttle, 2007; Rohwer \& Thurber, 2009; Letarov \& Kulikov, 2009; Rodriguez-Valera et al., 2009; Reyes et al., 2012; Brum \& Sullivan, 2015). In the oceans for example, viruses infecting cyanobacteria kill approximately 3\% of their hosts per day (Suttle, 2002), while also impacting cyanobacterial photosynthesis locally and globally through the expression and transfer of virus-encoded photosystem core genes (Lindell et al., 2005; Sullivan et al., 2006). Such modulation of host microbial metabolisms during infection appears to be a generalized strategy wherein oceanic viral communities encode genes with the potential to modulate key microbial carbon, nitrogen, phosphate and sulfur metabolisms (Breitbart et al., 2007; Sharon et al., 2009; Sharon et al., 2011; Thompson et al., 2011; Hurwitz, Hallam \& Sullivan, 2013; Anantharaman et al., 2014; Roux et al., 2014b; Hurwitz et al., 2015). In humans, viruses of microbes appear dynamic (Reyes et al., 2010; Pride et al., 2011; Minot et al., 2013), and again likely play key ecosystem roles, particularly affecting virulence of facultative pathogens (Boyd, 2012; Busby, Kristensen \& Koonin, 2013) with a striking example being the requirement of a phage infection for the full virulence of Vibrio cholerae (Waldor \& Mekalanos, 1996). Microbial viruses may also help fight antibiotic-resistant pathogens, leading to a recent resurgence in research exploring the use of viruses for "phage therapy" in humans (Bush et al., 2011; Nobrega et al., 2015).

In spite of this importance, our understanding of viral diversity remains limited to a tiny fraction of that occurring in nature. This is because most microbes known to exist from barcode surveys are not yet in culture (Rappé \& Giovannoni, 2003), and even if microbial hosts were cultivated, not all viruses are amenable to cultivation (Edwards \& Rohwer, 2005). In the oceans alone, the lack of reference genomes leads to surveys of viral communities returning mostly (63-93\%) unknown sequences (Brum \& Sullivan, 2015), and most (99\%) of 5,476 surface ocean viral populations remaining taxonomically unidentifiable beyond the "order" level (Brum et al., 2015). This is not surprising given that $86 \%$ of the 1,531 genomes of viruses that infect bacteria and archaea available at RefSeq are associated with only 3 of 61 known host phyla (based on the viral genomes available in NCBI RefseqVirus v69, January 2015).

One way forward is to better detect and catalog viral sequence data from rapidly expanding microbial genomic datasets. First, prophages, which result from the integration of a temperate virus genome into a microbial host genome, are present in $\sim 60 \%$ of sequenced bacteria (Casjens, 2003; Canchaya, Fournous \& Brüssow, 2004). Second, Single-cell Amplified Genome (SAG) datasets are now routinely generated to provide genome sequence data and inferences about metabolic capacity for novel microbes (Swan et al., 2011; Kamke, Sczyrba \& Ivanova, 2013; Rinke et al., 2013; Kashtan et al., 2014), and offer a rich source of novel viral sequences. These data will include prophage sequences, as well as viruses from actively lytic infections. Such SAG-based viral signal has already provided insights into marine viral diversity and virus-host interactions in uncultivated protists, bacteria and archea (Yoon et al., 2011; Roux et al., 2014b; Labonté et al., 2015). Third, large genome fragments of uncultivated microbes and associated viruses can now be assembled from microbial metagenomes (Sharon et al., 2009, 2011; Narasingarao et al., 2012; Albertsen et al., 2013; Anantharaman et al., 2014). Finally, viral metagenomics (viromics) can be used to survey the sequence data associated with purified viral particles and can also result in assembly of large viral genome fragments (Emerson et al., 2012; Minot et al., 2013; Roux et al., 2013; Brum et al., 2015).

Numerous approaches are available to identify prophages in complete microbial genomes 
including Phage_Finder (Fouts, 2006), Prophinder (Lima-Mendez et al., 2008), PHAST(Zhou et al., 2011), and PhiSpy (Akhter, Aziz \& Edwards, 2012). Overall, prophage predictors rely on the detection of sequence similarities between regions of the microbial genome and known viral genes. In addition, PhiSpy also identifies "viral-like" genomic features (AT and GC skew, protein length and transcription strand directionality) to enable the detection of viruses absent from databases (Akhter, Aziz \& Edwards, 2012). Prophage predictors also look for prophage "ends" by identifying the attachment sites in the microbial genome for each predicted prophage. These tools are either designed for a user to download and run locally (PhiSpy, Phage_Finder) or to access through a web-server (PHAST).

However, new tools are needed that (i) advance viral detection beyond prophages and instances where new viruses closely match those available in databases, and (ii) can handle fragmented and larger-scale microbial genomic datasets. Here, we present VirSorter, an automated tool designed to detect viral signal in genomic datasets, and make this new tool and the associated databases freely available in the Discovery Environment of the iPlant Cyberinfrastructure (Goff et al., 2011). Overall, we demonstrate that VirSorter detects prophages in complete microbial genomes as well as current prophage tools, but also offers capabilities to detect viral sequences in fragmented genomic datasets including incomplete genomes, SAGs or metagenomic assemblies, and can be used to flag potential cellular contamination in viromes for removal.

\section{Materials \& Methods}

\section{Building reference databases for bacterial and archaeal viruses}

Two reference databases of viral protein sequences were built for VirSorter and are available in the iPlant Discovery Environment (Data/Community_Data/iVirus/VirSorter/Database). The first includes 114,297 proteins from viruses infecting bacteria or archaea in RefSeqVirus genomes (as of January 2014), hereafter named "RefSeqABVir". Protein clusters (PCs) were defined using MCL clustering (Enright, Van Dongen \& Ouzounis, 2002) of these proteins (inflation 2.0) based on their reciprocal blastp comparisons (threshold of 50 on bit score and $10^{-03}$ on E-value). The 9,735 PCs with at least 3 sequences were used to define a profile database searchable with HMMER3 tools (Eddy, 2011). The remaining 34,668 unclustered sequences were formatted for a blastp search. All PCs that did not contain any sequences from Caudovirales and unclustered sequences from viruses other than Caudovirales were marked as "Non-Caudovirales".

The RefSeqABVir database was then augmented by virome sequences sampled from freshwater, seawater, and human gut, lung and saliva, resulting in an extended version of the reference database (hereafter named "Viromes") which includes both virome and RefSeqABVir sequences. This combined reference dataset should help to detect new viruses for which no cultivated reference sequence is available. When only raw reads were available, viromes were assembled using Newbler (threshold of $98 \%$ identity on $35 \mathrm{bp}$ ). The resulting contigs were then checked for the presence of cellular genome sequences, and only the 68 viromes for which no $16 \mathrm{~S}$ rRNA genes were retained (see Supplementary Table 1 for a complete list of these viromes). Contigs assembled from these 68 viromes were then manually inspected (through annotations generated by Metavir; Roux et al., 2014a) and revealed no identifiable cellular genome sequences (i.e. no sequence contained more than 2 genes that matched a cellular genome and were not found in any known virus). A total of 146,521 complete predicted proteins from this quality-controlled dataset were then clustered with the 114,297 proteins from RefSeqABVir, leading to 15,673 clusters with 3 sequences or more, and 88,052 unclustered sequences. PCs from the combined Viromes database were used to create a profile database searchable with HMMER3, and the 34,338 unclustered sequences from RefseqABVir were formatted for BLAST search (unclustered sequences from viromes were not added to the database to prevent the inclusion of 
contaminating sequences).

Within these databases, viral "hallmark" genes were defined though a text-searching script looking for "major capsid protein", "portal", "terminase large subunit", "spike", "tail", "virion formation" or "coat" annotations. After a manual curation step removing genes with more general annotation such as "protease" or "chaperone", 826 PCs or single genes were identified as "viral hallmark genes". This latter point meant removing domains also matching "protease" or "chaperone" domains and was conducted to minimize false positives for our viral hallmark genes category by extracautiously avoiding PCs that might include domains that could derive from either both viruses or microbes.

\section{VirSorter sequence pre-processing}

VirSorter was inspired by previous algorithms and tools developed to detect prophages (viral sequences integrated in cellular genomes), especially Prophinder (Lima-Mendez et al., 2008). For each (set of) genome(s) and/or contig(s) (for draft genomes) provided as raw nucleotide sequences, the initial stages of VirSorter include a detection of circular sequences (i.e. sequences with matching ends likely representing circular templates; Roux et al., 2014a), gene prediction on each sequence with MetageneAnnotator (Noguchi, Taniguchi \& Itoh, 2008), and selection of all sequences with more than 2 genes predicted. VirSorter also removes all poor-quality predicted protein sequences (predicted protein sequences with more than 50 consecutive X, F, A, K or P residues) likely originating from gene prediction across low-complexity or poorly defined genome regions (e.g. "bridges" between contigs generated during scaffolding) and yielding false-positive matches when compared to protein domain databases.

Predicted protein sequences are then compared to PFAM (v27) and to the viral database selected by the user (either RefSeqABVir or Viromes) with hmmsearch (Eddy, 2011) and blastp (Altschul et al., 1997) and each gene is affiliated to its most significant hit based on alignment score. Thresholds for significant hits are as follows: minimum score of 40 and maximum E-value of $10^{-05}$ for hmmsearch, and minimum score of 50 and maximum E-value of $10^{-03}$ for blastp.

\section{VirSorter metrics computation}

Following the sequence pre-processing, viral regions are detected through computation of multiple metrics using sliding windows. The metrics used are $(i)$ presence of viral hallmark genes (Koonin, Senkevich \& Dolja, 2006; Roux et al., 2014b), (ii) enrichment in viral-like genes (i.e. genes with best hit against the viral reference database, either RefSeqABVir or Viromes), (iii) depletion in PFAM affiliated genes, (iv) enrichment in uncharacterized genes (i.e. predicted genes with no hits either in PFAM or the viral reference database), $(v)$ enrichment in short genes (genes with a size within the $10 \%$ shorter genes of the genome), and ( $v i)$ depletion in strand switching (i.e. change of coding strand between two consecutive genes).

For all the enrichment and depletion metrics, a score comparable to the one of Prophinder was used (Lima-Mendez et al., 2008). First, a global value for each metric is estimated for the whole genome set (global rate of viral-like genes, global rate of PFAM-affiliated genes, etc). Then, for each window, the number of observed events (e.g. number of viral-like genes) is compared to an expected number deduced from the global value of the metric (modeled with a binomial law). A $p$-value is computed, reflecting the probability of observing $n$ events or more (for enrichment) or $n$ events or fewer (for depletion) at random, thus corresponding to a risk of generating false positives. These $p$-values are multiplied by the total number of comparisons (here the total number of sliding windows observed on a sequence), and a negative logarithmic transformation $\left(-\log _{10}\right)$ defines the associated significance score, again as in the Prophinder algorithm (Lima-Mendez et al., 2008). 


\section{Sequence metrics summary}

Each metric is computed using sliding windows from 10 to 100 genes wide, starting at every gene along the sequence, and all scores greater than 2 are stored. Local maxima of significance score are then searched and the associated set of genes is defined as a putative viral region. These different predictions (based on the metrics above) are then merged when overlapping (extending the regions to include all predicted windows), leading to a list of putative viral regions associated with a (set of) metric(s). These regions are classified into three categories: (i) category 1 ("most confident" predictions) regions have significant enrichment in viral-like genes or non-Caudovirales genes on the whole region and at least one hallmark viral gene detected; (ii) category 2 ("likely" predictions) regions have either enrichment in viral-like or non-Caudovirales genes, or a viral hallmark gene detected, associated with at least one other metric (depletion in PFAM affiliation, enrichment in uncharacterized genes, enrichment in short genes, depletions in strand switch); and (iii) category 3 ("possible" predictions) regions have neither a viral hallmark gene nor enrichment in viral-like or non-Caudovirales genes, but display at least two of the other metrics with at least one significance score greater than 4 . Finally, if a predicted region spans more than $80 \%$ of predicted genes on a contig, the entire contig is considered viral. A summary of VirSorter detection types is displayed in Figure 1B.

Next, higher confidence predictions are used to refine the sequence space search. Specifically, sequences from all open reading frames from category 1 predictions that do not match a viral protein cluster are clustered and added to the reference database (RefSeqABVir or Viromes depending on the initial user choice). This updated database is then used in another round of search by VirSorter. This iteration where category 1 sequences are used to refine the searches is continued until no new genes are added to the database. Once no new genes are added, the final VirSorter output is provided to the user and includes nucleotide sequences of all predicted viral sequences in fasta files, an automatic annotation of each prediction in genbank file format, and a summary table displaying for each prediction the associated category and significance scores of all metrics. By providing the predictions and the underlying significance scoring, users can evaluate each prediction and apply custom thresholds on significance scores through a simple text-parsing script, even for large-scale datasets.

VirSorter is available as an application (App) in the iPlant discovery environment (https://de.iplantcollaborative.org/de/) under Apps/Experimental/iVirus (see Supplementary Figure S1 for a step-by-step guide of VirSorter app on iPlant). This application allows users to search any set of contigs for viral sequences using either the RefSeqABVir or the Viromes database. The reference values of VirSorter metrics will be evaluated on the complete set of input sequences, hence mixed datasets should be sorted (when possible) by type of bacteria or archaea in order to get the most accurate result possible. In addition to these reference databases, the VirSorter App on iPlant allows users to input their own reference viral genome sequence already assembled or to-be assembled using 
209 iPlant Apps prior to analysis with VirSorter. Assembled sequences are processed as follows: $(i)$ genes 210 are predicted with MetaGeneAnnotator (Noguchi, Taniguchi \& Itoh, 2008), (ii) predicted proteins are 211 clustered with sequences from the user-selected database (either RefSeqABVir or Viromes), and (iii) 212 unclustered proteins are added to the "unclustered" pool. VirSorter scripts are also available through

\section{Comparison of VirSorter with other prophage predictors}

We first evaluated VirSorter results against the manually curated prophages from (Casjens, 2003). Each genome was processed with VirSorter, PhiSpy (Akhter, Aziz \& Edwards, 2012), Phage_Finder (Fouts, 2006) and PHAST (Zhou et al., 2011). For each tool, a prophage was considered as "detected" when a prediction covered more than $75 \%$ of the known prophage. For a more detailed example case of prophage detection in a complete bacterial genome including both prophages and genomic islands, the same tools were applied to the manually annotated Pseudomonas aeruginosa LES B58 genome (Winstanley et al., 2009).

VirSorter was then compared with the same prophage detection tools on the set of simulated SAGs. In that case, a viral sequence was considered as detected if predicted as completely viral or as a prophage. All the additional detections were manually checked to verify if the region was indeed viral (originating from a prophage in one of the microbial genomes rather than from a viral genome) or a false positive. The same approach was used for the simulated microbial and viral metagenomes results.

For each set of predictions, two metrics are computed. First, the Recall value corresponds to the number of viral sequences correctly predicted divided by the total number of known viral sequences in the dataset, and reflects the ability of the tool to find every known viral sequence in the dataset. Second, the Precision value is computed as the total number of viral sequences correctly predicted divided by the total number of viral sequences predicted, and indicates how accurate the tool is in its identification of viral signal.

Simulation of draft genomes and metagenomes.

A total of 10 Single-cell amplified genomes, 10 microbial metagenomes and 10 viral metagenomes were simulated with NeSSM (Jia et al., 2013). Microbial genomes were randomly picked within the bacterial and archaeal genomes availabe in RefSeq and WGS (as of January 2014). Viral genomes were picked within the most recently submitted genomes (since June 2014), thus are not in VirSorter reference database. Simulated inputs for each genome group (viral and microbial) followed a power-law distribution of abundances within the microbial and viral communities. The proportion of viral reads varied from 5 to $20 \%$ for microbial metagenome, and from 75 to $99 \%$ for viral metagenomes (Table S4 and Table S5). For each simulated dataset, 100bp paired-end reads similar to those obtained with HiSeq Illumina were generated (100,000 for SAGs, 1,000,000 for metagenomes), QC'd with fastq quality_trimmer with a threshold of 30 (part of the fastx_toolkit, http://hannonlab.cshl.edu/fastx_toolkit/), and assembled with Idba_ud (Peng et al., 2012).

To identify viral sequences in the assemblies, the resulting contigs were compared to the viral genomes with nucmer (Delcher, Salzberg \& Phillippy, 2003), and all sequences matching one of the viral genomes at $97 \%$ nucleotide identity or more were considered as viral. All simulated contigs and composition table (i.e. relative abundance of each genome in the simulated dataset) are available in the iPlant Discovery Environment alongisde VirSorter results for each of these simulated datasets (/iplant/home/shared/imicrobe/VirSorter/Benchmark_datasets and Benchmark_results respectively).

\section{Results \& Discussion}




\section{Reference-dependent and general genome features used to detect viruses}

VirSorter is designed to predict viral sequences in complete or fragmented genome sequence data from bacteria and archaea. Viral sequences are identified through a combination of "primary metrics" linked to the detection of significant similarities with known viral sequences and "secondary metrics" associated with viral-like genome structure (Fig. 1A). VirSorter first builds a probabilistic model for each metric using the microbial genomic data provided by the user (i.e. the complete genome or the entire contig dataset for draft genomes or metagenomes) that is then used as reference to calculate enrichment/depletion statistics. A "statistical enrichment in viral gene content" for a set of genes thus indicates that the region evaluated displays more viral-like genes than would be expected by chance alone based on the overall frequency of viral-like genes in the whole dataset. Viral-like genes are identified through comparison to RefSeq viral genomes ("RefSeqABVir" database hereafter) or to a custom database built from RefSeqABVir to which curated virome datasets were added to improve novel virus detection capabilities (hereafter "Viromes" database, Fig. 1A, Table S1). Through the VirSorter application (App) on iPlant, users can also add their own viral genome sequence(s) (in fasta format), which predicted protein will be added to the user-selected database (either RefSeqABVir or Viromes).

\section{Viral signal mining process}

Viral regions are predicted based on a summary of primary and secondary metrics evaluated on each genomic sequence. Each prediction is categorized from 1 to 3 in order of decreased confidence (Fig. 1 A \& B). Sequences for which the predicted viral region spans more than $80 \%$ of the contig length are considered as entirely viral. Biologically, we interpret these different categories as sequences similar to known viral references (category 1), sequences divergent from references with mostly genes yet to be detected in viral genomes or partial sequences lacking viral hallmark genes which may include defective prophages (category 2), and sequences or regions with a genome structure similar to viral genomes, but lacking any similarity to known viruses or viromes (category 3). These latter, category 3 predictions are thus essentially "aberrant" cellular genomic regions, and as such should be carefully examined as this category also routinely includes hypervariable microbial genomic islands and other mobile genetic elements in addition to novel viral sequences. However, we include category 3 predictions since when coupled to manual inspection, researchers can use these predictions to uncover novel biology, particularly when analyzing the small contigs and highly novel viruses likely to derive from fragmented draft genomes or SAGs.

\section{Virsorter prophage prediction is comparable to existing tools}

To evaluate VirSorter performances, we first examined its prophage prediction capability as compared to existing tools. Specifically, we used a set of 267 manually annotated prophages from 54 bacterial genomes (Casjens, 2003) to compare the prophage prediction performances of VirSorter, PhiSpy, Phage_Finder, and PHAST. We evaluate performance using two metrics: (i) "Recall", the number of viral regions detected divided by the total number of viral regions (also known as "Sensitivity") and (ii) "Precision", the number of correct predictions divided by the total number of predictions (also known as "Positive Predictive Value").

All of the tested prophage prediction tools perform well on these complete genome datasets as Recall values range from 64 to 85\%, and Precision values range from 74 to 93\% (Fig. 2 and Table S2). Two of the tools also associate their predictions with a level of confidence: PHAST predictions are noted as "intact", "incomplete", or "questionable" based on the number and type of phage genes detected, and VirSorter categorizes predictions as described above. To see how these confidence 
categories impacted results, we computed scores with and without the least confident predictions for both of these tools (Fig. 2). For PHAST, adding the questionable detections increased detection sensitivity (Recall increased from 70 to $84 \%$ ) without altering the Precision (both sets of predictions display a Precision of $83 \%$ ). Conversely, including the least confident category 3 predictions for VirSorter only slightly increased Recall (73 to $79 \%$ ), but did so at the cost of Precision (dropping from 93 to $72 \%$ ). Hence, for VirSorter, prophages predicted as category 3 from complete microbial genomes are prone to "false-positive" detections - notably because they can also include other genomic regions with unusual sequence composition features such as genomic islands or mobile genetic elements (see below).

Next, we focused on the case of prophages prediction in the manually annotated Pseudomonas aeruginosa LES B58 genome, which includes both prophages and genomic islands (Winstanley et al., 2009), to better explore how these tools deal with divergent prophages and unusual genomic regions (Table 1). All 6 known prophages in this genome were detected by VirSorter (categories 1 or 2), and PHAST (though 4 were considered "questionable"), whereas PhiSpy and Phage_Finder detected only 5 and 4, respectively. These missed prophages were the shortest ones (12 and 19 genes compared to $>40$ genes for all the other prophages), and one (Prophage_6) also corresponded to an unusual phage from the Inoviridae familiy, under-represented in viral genome databases. Beyond prophages, this microbial genome also displayed 5 manually curated genomic islands. None of these genomic islands were detected as a prophage by Phage_Finder, while PhiSpy and PHAST each wrongly identifies one of these genomic islands as a prophage, and VirSorter identifies two of them as category 3 predictions (i.e. putative prophage or other unusual genomic feature, Table 1). This example illustrates that category 3 predictions from VirSorter help capture even divergent prophages, but also detect hypervariable regions in microbial genomes, such as genomic islands or plasmids.

\section{VirSorter is more efficient at mining viral signal from single-cell amplified genomes (SAGs)}

To evaluate the capacity of prophage predictors and VirSorter to detect viral sequences in SAG datasets, we generated 10 simulated datasets of 100,000 reads (100bp) from one microbial and one viral genome, with 5 to $10 \%$ of the reads originating from the viral genome (Table S3). For each simulated dataset, reads were assembled into contigs (averages $=556$ contigs per SAG $\sim 3.3 \mathrm{~kb}$ in length), from which viral sequences or prophages were then predicted (the viral genomes used in the simulated datasets being absent from the VirSorter reference database).

On these SAGs, VirSorter outperformed all other tools as the only one maintaining comparable Recall and Precision values to those from complete microbial genomes (Fig. 2B). VirSorter categories $1 \& 2$ (higher confidence predictions) displayed a Recall of $65 \%$ and a Precision of $100 \%$, while adding in category 3 predictions increased Recall (88\%) but reduced Precision (81\%). Thus, for fragmented genomes, category 3 predictions help recover more viral sequences, but do so at the cost of increased false-positives. In comparison, PHAST (with or without the "questionable" predictions) performed at $40-50 \%$ Recall and 38-41\% Precision, whereas PhiSpy and Phage_Finder had a lower Recall (36 and 20\%, respectively) but high Precision (90 and 83\%, respectively). Considering that the prophage detection tools were optimized for viral sequence detection in complete microbial genomes, it is not surprising that VirSorter performs better for fragmented genomes.

We also applied VirSorter and the prophage predictors to a set of 127 SAGs from the uncultivated bacteria SUP05 for which viral sequences were previously manually identified and curated (Roux et al., 2014b). Of the 69 known viral contigs in this dataset, 62 were detected by VirSorter (including 29 as category 3), with the remaining 7 being too short (5.1 kb on average) to provide significant enrichment scores. In contrast, PHAST, PhiSpy and Phage_Finder detected only 15, 1 and none of these sequences, respectively. Beyond the fragmented nature of these SUP05 SAGs, 
these data likely represent a worst case scenario for the prophage prediction tools as these 69 SUP05

351 viruses represented new viral genera, and thus no closely related reference sequence were available in

\section{VirSorter alone is able to mine viral signal from bacterial and viral metagenomes}

We next evaluated VirSorter's capability to recover viral sequences in fragmented genomes assembled from metagenomic datasets. To this end, we created 10 'metagenomes' from 15 microbial and 15 viral genomes at varying representative abundances (Table S4 and see Methods). These simulated datasets total 192,941 contigs, so the scale is quite large - none of the prophage predictors were able to even process the data in a reasonable time (i.e. less than several days). Given that metagenome-derived contigs also represented fragmented genomes, we expect that performance would have been poor for prophage prediction tools on these datasets - likely even worse than the SAGs performance testing above.

VirSorter, however, was designed for and is thus able to handle such datasets. For contigs greater than 500 bp, VirSorter predictions displayed good Precision (93-100\%) but low Recall (33\%, Fig.3A, Supplementary Table 4). However, as the size of the contigs increased, Recall increased to 79$84 \%$ for contigs $>3 \mathrm{~kb}$ and $95-97 \%$ for contigs $>10 \mathrm{~kb}$, with no Precision loss (Fig. 3B).

Finally, we evaluated the potential of VirSorter to detect viral sequences in viral metagenomes contaminated with cellular genomes. Such cellular sequence in viral-fraction metagenomes can derive from co-purified encapsidated DNA (in gene transfer agents or generalized transducing phages) or contamination, and represents a common challenge in making inferences from viromes (Roux et al., 2013). We thus simulated 10 viral metagenomes of $1,000,000$ reads (100 bp) from a mix of 15 microbial and 60 viral genomes. This time, we simulated metagenomes where viral reads represented a larger proportion of the dataset, ranging from 75 to $99 \%$ (Table S5). Here, all microbial genomes available in RefSeq and WGS (as of January 2014) were used by VirSorter to model microbial genomic metrics instead of the whole dataset, since viromes largely lack microbial sequences. This usage case of VirSorter is implemented in the iPlant application and is available by checking the box "virome decontamination" in the submission form.

As found above for prediction of viral sequence data from the microbial metagenome simulations, VirSorter performance as a 'virome decontaminator' improves as contig size increases (Table 2, Table S5). When considering all contigs (>500 bp), the Recall of viral sequences is $32 \%$ on average, but increases up to $86 \%$ for contigs $>3 \mathrm{~kb}$ and $97 \%$ for contigs $>10 \mathrm{~kb}$. When category 3 predictions are included, these Recall values increase slightly to $33 \%, 90 \%$ and $99.8 \%$ for the increasing contig sizes, respectively. At the same time, the Precision of viral sequence detection stays high for all contig sizes, even when including category 3 predictions ( $99 \%$ and more, Table 2$)$.

\section{VirSorter's strengths and weaknesses}

VirSorter represents a novel, scalable, and community-available tool for detecting and identifying viral genome sequences from diverse microbial datasets. Its performance for prophage prediction is largely comparable to that of available prophage prediction tools when applied to complete microbial genomes, but it outperforms available tools when making predictions from modern microbial datasets which tend to be fragmented and larger-scale or when searching for viruses beyond those "known" in current databases. Thus, VirSorter complements existing tools to help elucidate bacterial and archaeal viral sequences among myriad modern microbial genomic data types.

However, VirSorter does have limitations. First, VirSorter was designed and optimized for detection of bacterial and archaeal viruses, so it does not detect eukaryotic viruses well. This is because the database lacks eukaryote viruses, and the viral genome features were only evaluated on bacterial 
and archaeal viruses. VirSorter will still detect eukaryote viruses, often as category 3 because of their singular genome composition (compared to a typical cellular genome), but its capacity is extremely limited in its current build. Second, short $(<3 \mathrm{~kb})$ viral contigs will tend to only be detected by VirSorter when they contain hallmark genes. Pragmatically, this means that viral signal detection in non-assembled reads or in contigs assembled from (meta)transcriptome data will usually be inefficient. Third, prophage prediction tools also look for additional signs of prophages such as the presence of integrase genes, att sites, or repeat features to demarcate the 'ends' of a prophage genome, none of these features are examined by VirSorter. Thus, prophage prediction tools likely remain the best means to most accurately annotate prophages in a complete microbial genome, whereas VirSorter is best used for high-throughput analyses and for detecting viral signal in fragmented genomes. Finally, category 3 detections represent sequences and regions that are unique within the genome(s) being compared, so while many can be viral, these predictions could also represent other mobile genetic elements or hypervariable genomic islands and require manual curation. The only case where category 3 predictions may be considered without manual curation are viral metagenome decontaminations as these predictions increase Recall while only marginally lowering Precision.

\section{Acknowledgements}

We thank Nirav Merchant, Darren Boss, and Ken Youens-Clark for aiding in setting up VirSorter on the iPlant platform, Rachel Whitaker and Whitney England for their assistance with Pseudomonas aeruginosa LES B58 genome annotation, as well as TMPL members for comments on the manuscript.

\section{References}

Akhter S, Aziz RK, Edwards RA. 2012. PhiSpy: a novel algorithm for finding prophages in bacterial genomes that combines similarity- and composition-based strategies. Nucleic Acids Research 40:1-13.

Albertsen M, Hugenholtz P, Skarshewski A, Nielsen KL, Tyson GW, Nielsen PH. 2013. Genome sequences of rare, uncultured bacteria obtained by differential coverage binning of multiple metagenomes. Nature Biotechnology 31:533-8. DOI: 10.1038/nbt.2579.

Altschul SF, Madden TL, Schäffer a a, Zhang J, Zhang Z, Miller W, Lipman DJ. 1997. Gapped BLAST and PSI-BLAST: a new generation of protein database search programs. Nucleic Acids Research 25:3389-402.

Anantharaman K, Duhaime MB, Breier JA, Wendt K, Toner BM, Dick GJ. 2014. Sulfur Oxidation Genes in Diverse Deep-Sea Viruses. Science. DOI: 10.3354/meps145269.

Boyd EF. 2012. Bacteriophage-encoded bacterial virulence factors and phage-pathogenicity island interactions. In: Łobocka M \& Szybalski WT ed. Advances in Virus Research, Vol. 82. Elsevier Inc., 91-118. DOI: 10.1016/B978-0-12-394621-8.00014-5.

Breitbart M, Thompson LR, Suttle CA, Sullivan MB. 2007. Exploring the Vast Diversity of Marine Viruses. Oceanography 20:135-139. 
Breitbart M, Rohwer F. 2005. Here a virus, there a virus, everywhere the same virus? Trends in Microbiology 13:278-284.

Brum JR, Ignacio-espinoza JC, Roux S, Doulcier G, Acinas SG, Alberti A, Chaffron S, Coppola L, Cruaud C, de Vargas C, Gasol JM, Gorsky G, Gregory AC, Guidi L, Hingamp P, Iudicone D, Not F, Ogata H, Pesant S, Poulos BT, Schwenck SM, Speich S, Dimier C, Picheral M, Searson S, Kandels-Lewis S, Coordinators TO, Bork P, Bowler C, Karsenti E, Sunagawa S, Wincker P, Sullivan MB. 2015. Global patterns and ecological drivers of ocean viral communities. Science in press.

Brum JR, Sullivan MB. 2015. Rising to the challenge: accelerated pace of discovery transforms marine virology. Nature Reviews Microbiology 13:147-59. DOI: 10.1038/nrmicro3404.

Busby B, Kristensen DM, Koonin E V. 2013. Contribution of phage-derived genomic islands to the virulence of facultative bacterial pathogens. Environmental Microbiology 15:307-312. DOI: 10.1111/j.1462-2920.2012.02886.x.

Bush K, Courvalin P, Dantas G, Davies J, Eisenstein B, Huovinen P, Jacoby G a, Kishony R, Kreiswirth BN, Kutter E, Lerner S a, Levy S, Lewis K, Lomovskaya O, Miller JH, Mobashery S, Piddock LJ V, Projan S, Thomas CM, Tomasz A, Tulkens PM, Walsh TR, Watson JD, Witkowski J, Witte W, Wright G, Yeh P, Zgurskaya HI. 2011. Tackling antibiotic resistance. Nature Reviews Microbiology 9:894-6. DOI: 10.1038/nrmicro2693.

Canchaya C, Fournous G, Brüssow H. 2004. The impact of prophages on bacterial chromosomes. Molecular Microbiology 53:9-18. DOI: 10.1111/j.1365-2958.2004.04113.x.

Casjens S. 2003. Prophages and bacterial genomics: what have we learned so far? Molecular Microbiology 49:277-300. DOI: 10.1046/j.1365-2958.2003.03580.x.

Delcher AL, Salzberg SL, Phillippy AM. 2003. Using MUMmer to identify similar regions in large sequence sets. Current Protocols in Bioinformatics Chapter 10:Unit 10.3. DOI: 10.1002/0471250953.bi1003s00.

Eddy SR. 2011. Accelerated Profile HMM Searches. PLoS Computational Biology 7:e1002195. DOI: 10.1371/journal.pcbi.1002195.

Edwards RA, Rohwer F. 2005. Viral metagenomics. Nature Reviews Microbiology 3:504-510.

Emerson JB, Thomas BC, Andrade K, Allen EE, Heidelberg KB, Banfield JF. 2012. Metagenomic assembly reveals dynamic viral populations in hypersaline systems. Applied and Environmental Microbiology 78:6309 - 6320. DOI: 10.1128/AEM.01212-12.

Enright AJ, Van Dongen S, Ouzounis CA. 2002. An efficient algorithm for large-scale detection of protein families. Nucleic Acids Research 30:1575-84.

Fouts DE. 2006. Phage_Finder: automated identification and classification of prophage regions in 
complete bacterial genome sequences. Nucleic Acids Research 34:5839-51. DOI: $10.1093 / \mathrm{nar} / \mathrm{gk1732}$.

Fuhrman JA. 1999. Marine viruses and their biogeochemical and ecological effects. Nature 399:541548. DOI: $10.1038 / 21119$.

Goff SA, Vaughn M, McKay S, Lyons E, Stapleton AE, Gessler D, Matasci N, Wang L, Hanlon M, Lenards A, Muir A, Merchant N, Lowry S, Mock S, Helmke M, Kubach A, Narro M, Hopkins N, Micklos D, Hilgert U, Gonzales M, Jordan C, Skidmore E, Dooley R, Cazes J, McLay R, Lu Z, Pasternak S, Koesterke L, Piel WH, Grene R, Noutsos C, Gendler K, Feng X, Tang C, Lent M, Kim S-J, Kvilekval K, Manjunath BS, Tannen V, Stamatakis A, Sanderson M, Welch SM, Cranston K a, Soltis P, Soltis D, O'Meara B, Ane C, Brutnell T, Kleibenstein DJ, White JW, Leebens-Mack J, Donoghue MJ, Spalding EP, Vision TJ, Myers CR, Lowenthal D, Enquist BJ, Boyle B, Akoglu A, Andrews G, Ram S, Ware D, Stein L, Stanzione D. 2011. The iPlant Collaborative: Cyberinfrastructure for Plant Biology. Frontiers in Plant Science 2:34. DOI: 10.3389/fpls.2011.00034.

Hurwitz BL, Brum JR, Sullivan MB. 2015. Depth-stratified functional and taxonomic niche specialization in the "core" and "flexible" Pacific Ocean Virome. The ISME Journal 9:472-84. DOI: 10.1038/ismej.2014.143.

Hurwitz BL, Hallam SJ, Sullivan MB. 2013. Metabolic reprogramming by viruses in the sunlit and dark ocean. Genome Biology 14:R123. DOI: 10.1186/gb-2013-14-11-r123.

Jia B, Xuan L, Cai K, Hu Z, Ma L, Wei C. 2013. NeSSM: a Next-generation Sequencing Simulator for Metagenomics. PloS One 8:e75448. DOI: 10.1371/journal.pone.0075448.

Kamke J, Sczyrba A, Ivanova N. 2013. Single-cell genomics reveals complex carbohydrate degradation patterns in poribacterial symbionts of marine sponges. The ISME Journal 7:2287300 .

Kashtan N, Roggensack SE, Rodrigue S, Thompson JW, Biller SJ, Coe A, Ding H, Marttinen P, Malmstrom RR, Stocker R, Follows MJ, Stepanauskas R, Chisholm SW, Biller J. 2014. Singlecell genomics reveals hundreds of coexisting subpopulations in wild Prochlorococcus. Science 344:416-20. DOI: 10.1126/science.1248575.

Koonin E V, Senkevich TG, Dolja V V. 2006. The ancient Virus World and evolution of cells. Biology Direct 1:29. DOI: 10.1186/1745-6150-1-29.

Labonté JM, Swan BK, Poulos BT, Luo H, Koren S, Hallam SJ, Sullivan MB, Woyke T, Wommack EK, Stepanauskas R. 2015. Single cell genomics-based analysis of virus-host interactions in marine surface bacterioplankton. The ISME Journal.

Letarov A, Kulikov E. 2009. The bacteriophages in human- and animal body-associated microbial communities. Journal of Applied Microbiology 107:1-13. DOI: 10.1111/j.1365- 
Lima-Mendez G, Van Helden J, Toussaint A, Leplae R. 2008. Prophinder: a computational tool for prophage prediction in prokaryotic genomes. Bioinformatics 24:863-5. DOI: 10.1093/bioinformatics/btn043.

Lindell D, Jaffe JD, Johnson ZI, Church GM, Chisholm SW. 2005. Photosynthesis genes in marine

Minot S, Bryson A, Chehoud C, Wu GD, Lewis JD, Bushman FD. 2013. Rapid evolution of the human viruses yield proteins during host infection. Nature 438:86-9. DOI: 10.1038/nature04111. gut virome. Proceedings of the National Academy of Sciences of the United States of America 110:12450-55. DOI: 10.1073/pnas.1300833110

Narasingarao P, Podell S, Ugalde J a, Brochier-Armanet C, Emerson JB, Brocks JJ, Heidelberg KB, Banfield JF, Allen EE. 2012. De novo metagenomic assembly reveals abundant novel major lineage of Archaea in hypersaline microbial communities. The ISME Journal 6:81-93. DOI: 10.1038/ismej.2011.78.

Nobrega FL, Costa AR, Kluskens LD, Azeredo J. 2015. Revisiting phage therapy: new applications for old resources. Trends in Microbiology:1-7. DOI: 10.1016/j.tim.2015.01.006.

Noguchi H, Taniguchi T, Itoh T. 2008. MetaGeneAnnotator: detecting species-specific patterns of ribosomal binding site for precise gene prediction in anonymous prokaryotic and phage genomes. DNA Research 15:387-96. DOI: 10.1093/dnares/dsn027.

Peng Y, Leung HCM, Yiu SM, Chin FYL. 2012. IDBA-UD: a de novo assembler for single-cell and metagenomic sequencing data with highly uneven depth. Bioinformatics 28:1420-1428.

Pride DT, Salzman J, Haynes M, Rohwer F, Davis-Long C, White RA, Loomer P, Armitage GC, Relman DA. 2011. Evidence of a robust resident bacteriophage population revealed through analysis of the human salivary virome. The ISME Journal 6:915-26. DOI: 10.1038/ismej.2011.169.

Rappé MS, Giovannoni SJ. 2003. The uncultured microbial majority. Annual Review of Microbiology 57:369-94. DOI: 10.1146/annurev.micro.57.030502.090759.

Reyes A, Haynes M, Hanson N, Angly FE, Heath AC, Rohwer F, Gordon JI. 2010. Viruses in the faecal microbiota of monozygotic twins and their mothers. Nature 466:334-38.

Reyes A, Semenkovich NP, Whiteson K, Rohwer F, Gordon JI. 2012. Going viral: next-generation sequencing applied to phage populations in the human gut. Nature Reviews Microbiology 10:60717. DOI: $10.1038 /$ nrmicro2853.

Rinke C, Schwientek P, Sczyrba A, Ivanova NN, Anderson IJ, Cheng J-F, Darling A, Malfatti S, Swan BK, Gies E a, Dodsworth J a, Hedlund BP, Tsiamis G, Sievert SM, Liu W-T, Eisen J a, Hallam SJ, Kyrpides NC, Stepanauskas R, Rubin EM, Hugenholtz P, Woyke T. 2013. Insights into the 
phylogeny and coding potential of microbial dark matter. Nature 499:431-7. DOI: 10.1038/nature12352.

Rodriguez-Valera F, Martin-Cuadrado A-B, Rodriguez-Brito B, Pasić L, Thingstad TF, Rohwer F, Mira A. 2009. Explaining microbial population genomics through phage predation. Nature Reviews Microbiology 7:828-36.

Rohwer F, Thurber RV. 2009. Viruses manipulate the marine environment. Nature 459:207-212.

Roux S, Krupovic M, Debroas D, Forterre P, Enault F. 2013. Assessment of viral community functional potential from viral metagenomes may be hampered by contamination with cellular sequences. Open Biology 3:130160.

Roux S, Tournayre J, Mahul A, Debroas D, Enault F. 2014a. Metavir 2: new tools for viral metagenome comparison and assembled virome analysis. BMC Bioinformatics 15:1-12.

Roux S, Hawley AK, Torres Beltran M, Scofield M, Schwientek P, Stepanauskas R, Woyke T, Hallam SJ, Sullivan MB. 2014b. Ecology and evolution of viruses infecting uncultivated SUP05 bacteria as revealed by single-cell- and meta- genomics. eLife 3:1-20. DOI: 10.7554/eLife.03125.

Sharon I, Alperovitch A, Rohwer F, Haynes M, Glaser F, Atamna-Ismaeel N, Pinter RY, Partensky F, Koonin E V, Wolf YI, Nelson N, Béjà O. 2009. Photosystem I gene cassettes are present in marine virus genomes. Nature 461:258-62. DOI: 10.1038/nature08284.

Sharon I, Battchikova N, Aro E-M, Giglione C, Meinnel T, Glaser F, Pinter RY, Breitbart M, Rohwer F, Béjà O. 2011. Comparative metagenomics of microbial traits within oceanic viral communities. The ISME journal 5:1178-90. DOI: 10.1038/ismej.2011.2.

Sullivan MB, Lindell D, Lee JA, Thompson LR, Bielawski JP, Chisholm SW. 2006. Prevalence and Evolution of Core Photosystem II Genes in Marine Cyanobacterial Viruses and Their Hosts. PLoS Biology 4:e234.

Suttle C. 2002. Cyanophages and their role in the ecology of cyanobacteria. In: Whitton BA \& Potts M ed. The ecology of cyanobacteria. Dordrecht: Springer Netherlands, 564-84.

Suttle CA. 2007. Marine viruses--major players in the global ecosystem. Nature Reviews Microbiology 5:801-812.

Swan BK, Martinez-Garcia M, Preston CM, Sczyrba A, Woyke T, Lamy D, Reinthaler T, Poulton NJ, Masland EDP, Gomez ML, Sieracki ME, DeLong EF, Herndl GJ, Stepanauskas R. 2011. Potential for chemolithoautotrophy among ubiquitous bacteria lineages in the dark ocean. Science 333:1296-300. DOI: 10.1126/science.1203690.

Thompson LR, Zeng Q, Kelly L, Huang KH, Singer AU, Stubbe J, Chisholm SW. 2011. Phage auxiliary metabolic genes and the redirection of cyanobacterial host carbon metabolism. Proceedings of the National Academy of Sciences of the United States of America 108:E757-64. 
DOI: 10.1073/pnas.1102164108.

575 Waldor MK, Mekalanos JJ. 1996. Lysogenic conversion by a filamentous phage encoding cholera toxin. Science 272:1910-1914.

577 Weinbauer MG. 2004. Ecology of prokaryotic viruses. FEMS Microbiology Reviews 28:127-81. DOI:

$578 \quad$ 10.1016/j.femsre.2003.08.001.

579 Winstanley C, Langille MGI, Fothergill JL, Kukavica-Ibrulj I, Paradis-Bleau C, Sanschagrin F, 580 Thomson NR, Winsor GL, Quail M a, Lennard N, Bignell A, Clarke L, Seeger K, Saunders D, 581 Harris D, Parkhill J, Hancock REW, Brinkman FSL, Levesque RC. 2009. Newly introduced genomic prophage islands are critical determinants of in vivo competitiveness in the Liverpool Epidemic Strain of Pseudomonas aeruginosa. Genome Research 19:12-23. DOI:

585 Wommack KE, Colwell RR. 2000. Virioplankton: viruses in aquatic ecosystems. Microbiology and Molecular Biology Reviews 64:69-114.

587 Yoon HS, Price DC, Stepanauskas R, Rajah VD, Sieracki ME, Wilson WH, Yang EC, Duffy S, 588 Bhattacharya D. 2011. Single-cell genomics reveals organismal interactions in uncultivated marine protists. Science 332:714-7. DOI: 10.1126/science.1203163.

590 Zhou Y, Liang Y, Lynch KH, Dennis JJ, Wishart DS. 2011. PHAST: a fast phage search tool. Nucleic Acids Research 39:W347-52. DOI: 10.1093/nar/gkr485. 
VirSorter process: overview (A) and examples of viral sequence detection (B).

(A) Overview of VirSorter process. The top part described the different parts of the sequence analysis pipeline, and the bottom frame summarizes the classification in three categories of decreasing confidence based on the different metrics being significant (green dot) or not (black cross). Viral "hallmark" genes or protein clusters (PCs) were identified by looking for genes typically of viral origin that are annotated as "major capsid protein", "portal", "terminase large subunit", "spike", "tail", "virion formation" or "coat" and manually removing all protein domains with a potential overlap with microbial functions. (B) Examples of viral sequence detection by VirSorter. On top is the clearest case, in which a sequence harbors several viral hallmark genes as well as enrichment in viral-like genes (or virome-like when the genes are most similar to a viral metagenome sequence, when using the Viromes database). This type of detection is considered as the most confident. The three examples below are different cases in which only one of the primary metrics is significant. Notably, these examples display how VirSorter can detect new viruses based on a significant depletion in characterized genes associated with a viral hallmark gene (case 3), and how the same number of genes can be a non-significant enrichment when considering all viruses, yet significant when looking at only the non-Caudovirales (case 4). These detections are still considered confident, although less sure than case 1. Finally, a last example (case 5) displays a more ambiguous situation, in which a sequence displays only secondary viral metrics but neither viral gene enrichment nor a viral hallmark gene. For these detections, one of the metrics (at least) must have an E-value lower than $10^{-04}$ (note that significance scores used in VirSorter output files are computed as negative $\log _{10}$ transformations of E-values, and would here correspond to a score of 4 or more). 
A

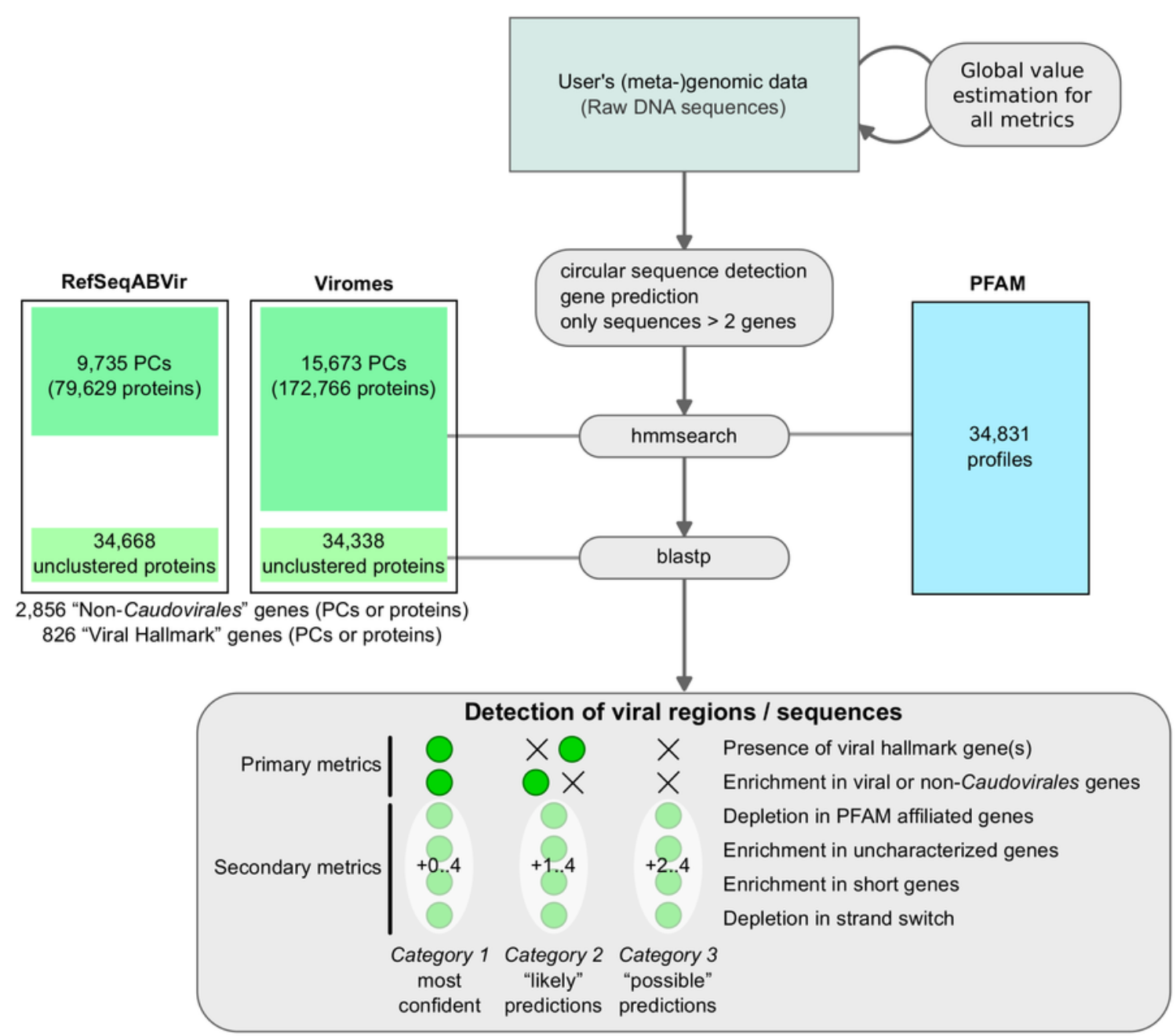

B
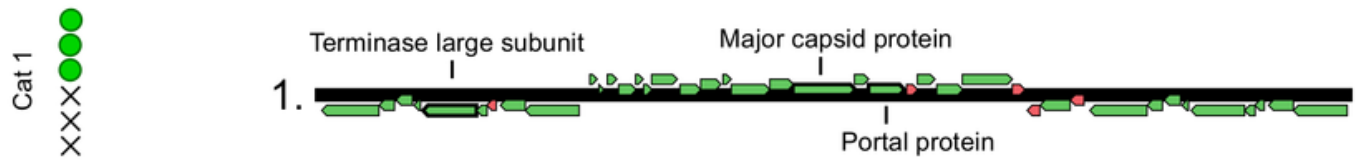

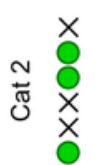

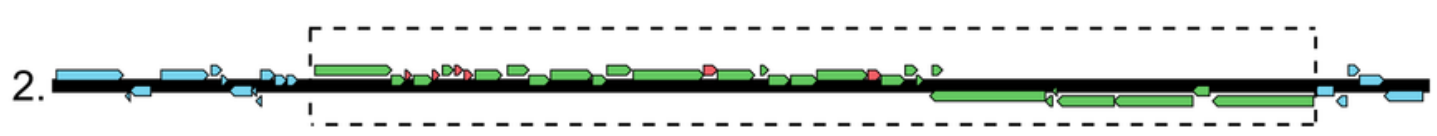

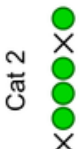

Major capsid protein

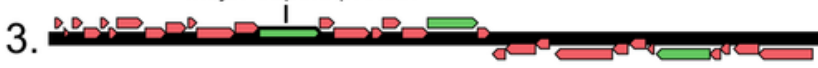

Viral- or Virome-like gene

Non-Caudovirales gene

$\square$ PFAM affiliated gene

$\stackrel{x}{\stackrel{x}{*}}$

Non-Caudovirales

$4 . \square$

Uncharacterized gene
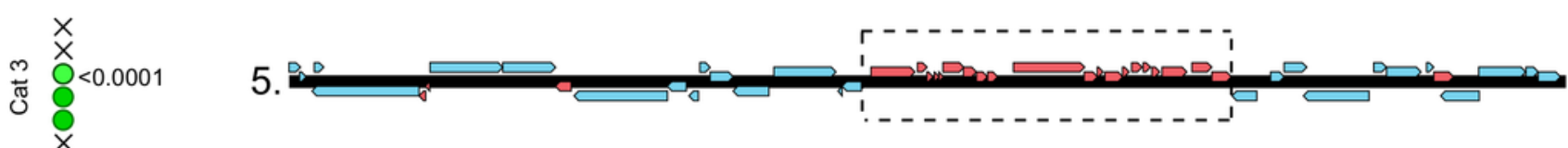
2

Accuracy of viral sequence predictions of VirSorter, PHAST, Phage_finder and PhiSpy on (A) complete microbial genomes, and (B) draft genomes from simulated SAGs including a microbial and viral genome.

For each set of predictions (i.e. each tool and set of option when applicable), the two metrics used to evaluate the tool performance are Recall ( $\mathrm{x}$-axis, proportion of known viral sequences or regions detected) and Precision (y-axis, proportion of predictions that corresponded to known viral sequences or regions). Prophages identified in the complete microbial genomes are compared to the list of manually curated prophages from Casjens, 2003. 
A

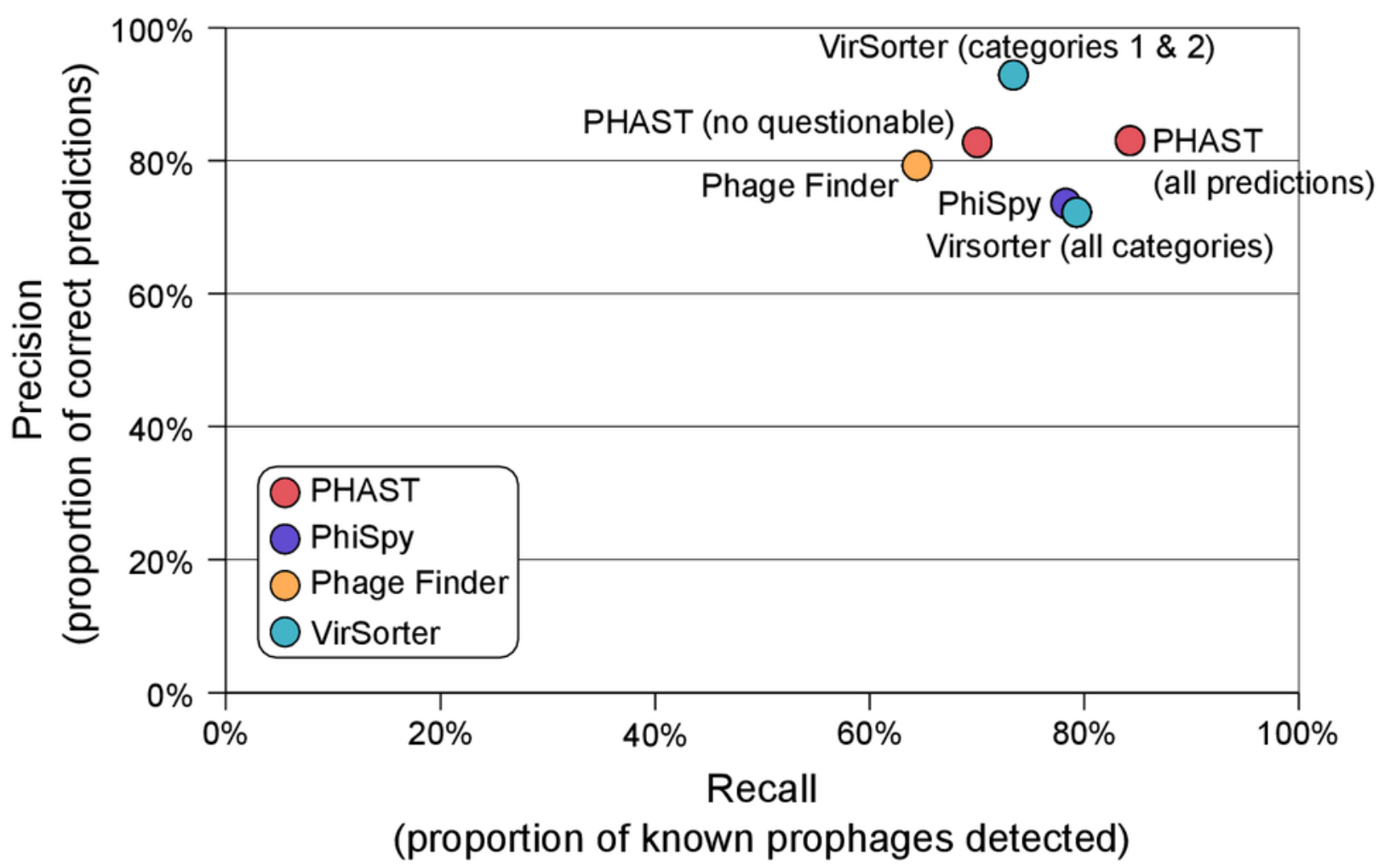

B

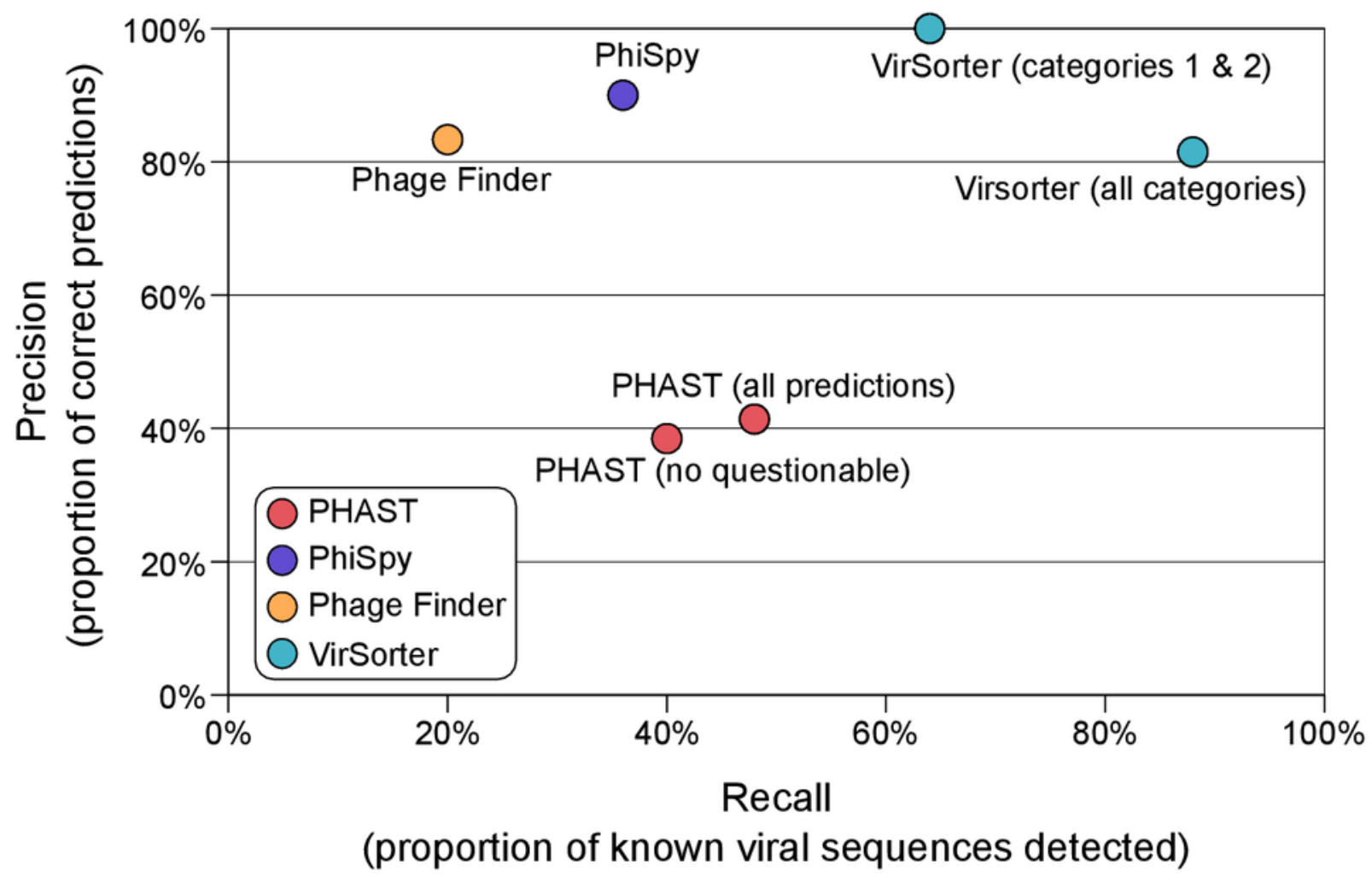




\section{3}

Figure 3: Detection of viral sequences in microbial metagenomes by VirSorter

(A) Average Recall ( $x$-axis) and Precision ( $y$-axis) of viral sequence detection by VirSorter in 10 simulated microbial metagenomes for different contig size thresholds. (B) Detection of viral sequences by VirSorter in simulated microbial metagenomes by contig size fraction. 
A

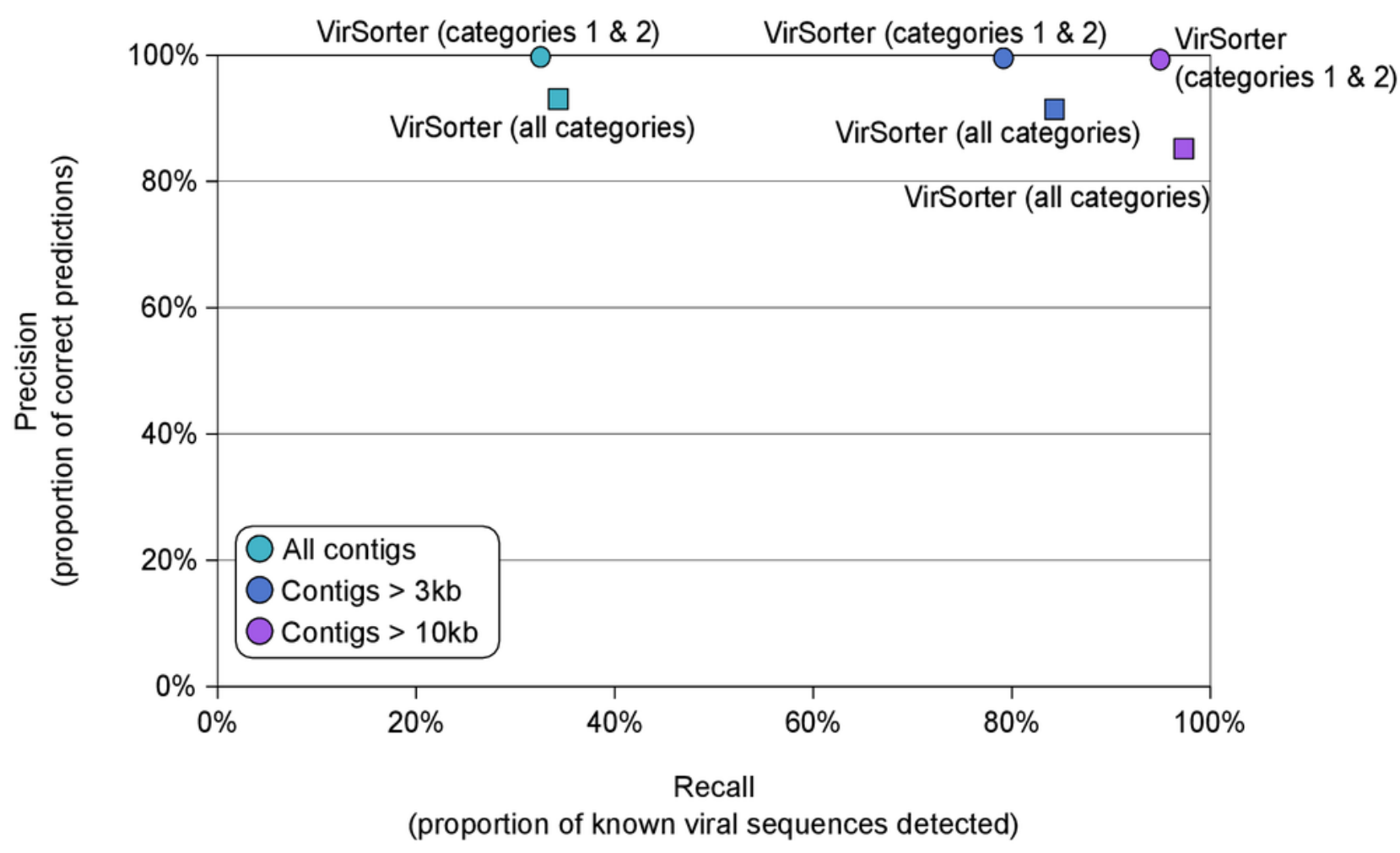

B

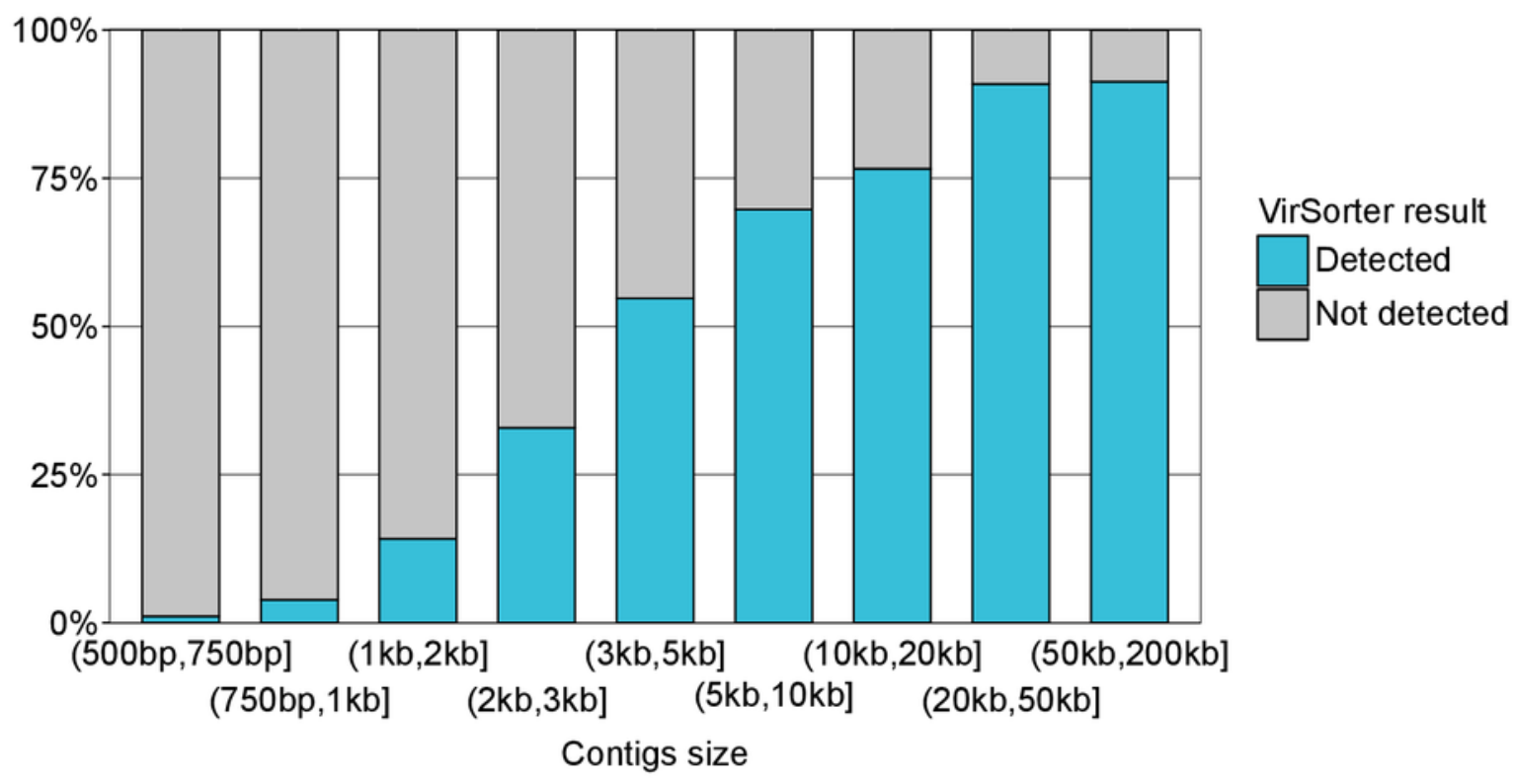


Table 1 (on next page)

Comparison of VirSorter predictions with prophage predictors on Pseudomonas aeruginosa LES B58 genome (NC_011770).

The coordinates of each prophage known on Pseudomonas aeruginosa LES B58 genome and detection for the different tools are indicated, with absence of detection highlighted in red. For VirSorter and PHAST, the category of detection (1, 2 or 3 for VirSorter, intact, incomplete or questionable for PHAST) is also indicated. False-positive detections of genomic islands as putative prophages are highlighted in orange. 


\begin{tabular}{cccccc}
\hline Feature & Coordinates & VirSorter & PHAST & PhiSpy & Phage_Finder \\
\hline Prophage 1 & $665,272-680,608$ & Prophage -2 & Prophage - questionable & Prophage & - \\
Prophage 2 & $863,875-906,018$ & Prophage -2 & Prophage - questionable & Prophage & Prophage \\
Prophage 3 & $1,433,756-1,476,547$ & Prophage -2 & Prophage - questionable & Prophage & Prophage \\
Prophage 4 & $1,684,045-1,720,850$ & Prophage -2 & Prophage - questionable & Prophage & Prophage \\
Genomic Island 1 & $2,504,700-2,551,100$ & Prophage -3 & Prophage - questionable & - & - \\
Prophage 5 & $2,690,450-2,740,350$ & Prophage -1 & Prophage - intact & Prophage & Prophage \\
Genomic Island 2 & $2,751,800-2,783,500$ & - & - & - & - \\
Genomic Island 3 & $2,796,836-2,907,406$ & - & - & Prophage & - \\
Genomic Island 4 & $3,392,800-3,432,228$ & - & - & - & - \\
Prophage 6 & $4,545,190-4,552,788$ & Prophage -2 & Prophage - intact & - & - \\
Genomic Island 5 & $4,931,528-4,960,941$ & Prophage -3 & - & - & - \\
\hline
\end{tabular}


Table 2 (on next page)

Results of VirSorter viral sequence detection on simulated viral metagenomes with a limited contamination by cellular genomes ( 1 to $25 \%$ of raw reads).

Metrics presented are Recall (proportion of viral sequences detected) and Precision (proportion of predictions corresponding to viral sequences). 


\section{VirSorter - categories 1 \& $2 \quad$ VirSorter - all categories}

\begin{tabular}{rcccc} 
& Recall & Precision & Recall & Precision \\
\hline All contigs $(>\mathbf{5 0 0} \mathbf{b p )})$ & $31.71 \%$ & $99.89 \%$ & $32.96 \%$ & $99.79 \%$ \\
Contigs $>\mathbf{3 k b}$ & $85.64 \%$ & $99.80 \%$ & $90.29 \%$ & $99.62 \%$ \\
Contigs $>\mathbf{1 0 k b}$ & $97.14 \%$ & $99.48 \%$ & $99.82 \%$ & $98.99 \%$ \\
\hline
\end{tabular}

3

4 